Natural Hazards and Earth System Sciences (2003) 3: 237-242

(C) European Geosciences Union 2003

(E) Natural Hazards

\title{
On the nature, scaling and spectral properties of pre-seismic ULF signals
}

\author{
F. Vallianatos ${ }^{1}$ and A. Tzanis ${ }^{2}$ \\ ${ }^{1}$ Department of Natural Resources Engineering, Technological Educational Institute of Crete, Chania Branch, Crete, Greece \\ ${ }^{2}$ Department of Geophysics and Geothermy, University of Athens, Panepistimiopoli, 15784 Zografou, Greece
}

Received: 1 July 2002 - Revised: 12 November 2002 - Accepted: 13 November 2002

\begin{abstract}
Earlier work by the authors (Vallianatos and Tzanis, 1999b), has proposed a model for the propagation and scaling of electric earthquake precursors, according to which the pre-seismic electric field emission is due to some time dependent polarisation appearing in an ensemble of electrified crustal volumes within the seismogenic source, which are distributed according to a fractal power law. Herein, we extend this formulation to the analysis of ULF magnetic precursors. We calculate the resulting transient magnetic field, which turns out to be mainly vertical and observable only if the seismogenic process generates a source with polarization rate perpendicular to the vertical plane through the source and the receiver. Furthermore, a scaling law between the vertical magnetic field and the magnitude of the associated earthquake is provided. We also investigate the spectral distribution law expected from such a set of emitters. To this effect, we assume that the evolution of the precursory polarisation process is quasi-incoherent over the exited ensemble, i.e. there is no unique relaxation time, but rather a spectrum of these with energy dependence expressed by an Arrhenius law with uniformly distributed energies. We show that the macroscopic ULF field resulting from the superposition of such an ensemble of sources has a power density spectrum distributed proportionally to $1 / f$. The above theoretical prediction appears to be consistent with independent observations by other investigators.
\end{abstract}

\section{Introduction}

During the past decade, a mounting body of evidence indicates that the study of electromagnetic phenomena associated with the preparation of large earthquakes is a promising field of earthquake prediction research (e.g. Hayakawa and Fujinawa, 1994; Hayakawa, 1999; Hayakawa and Molchanov, 2002). Among those, the observation and analysis of ULF magnetic fields is particularly encouraging, inasmuch as it

Correspondence to: A. Tzanis (atzanis@geol.uoa.gr) appears that credible case-studies of seismoelectromagnetic ULF emissions have been reported in the recent literature.

Well known are the earlier observations and models by Fraser-Smith et al. (1990), Molchanov et al. (1992), Park et al. (1993) and Fenoglio et al. (1995). Dea and Boerner (1999) report evidence of lithospheric ULF and ELF magnetic activity at a distance of $160 \mathrm{~km}$ to the south of the 17 January 1994, M6.7 Northridge, California (US) earthquake, commencing two weeks prior to the event. Conversely, Pilipenko et al. (1999) fail to detect any anomalies prior to the great Kobe earthquake (16 January 1995, $M_{S}=7.2$ ), at a distance of $400 \mathrm{~km}$ from the epicentre. Additional, more recent studies can be found in the first chapter of Hayakawa and Molchanov (2002).

In a series of in-depth investigations, Kopytenko et al. (1993, 1994a, b), Hayakawa et al. (1996), Kawata et al. (1998) and Hattori et al. (2002) among others, have observed a possible precursory signature in ULF electromagnetic data whose distinguishing feature was the high polarisation ratio of the vertical over the horizontal magnetic field components $(Z / H \geq 1)$, whereas typical magnetospheric ULF emissions exhibit low $Z / H$ ratios. This means that the presumed precursory ULF magnetic fields were overall vertically polarised. In another interesting development, Hayakawa et al. (1999) and Smirnova et al. (2001) have also argued that the lithospheric ULF magnetic fields recorded prior to the Guam earthquake (8 August 1993, $M_{S}=8$ ), evolved towards the structure of flicker noise (to be precise the power spectrum of ULF time series exhibited a powerlaw spectral dependence of the form $1 / f^{\beta}$, with $\beta$ decreasing toward unity as a function of the time-to-failure). Notably, flicker noise spectral behaviour is an expression of selforganized critical dynamics (Bak et al., 1987, 1988).

In conclusion, there exists not only compelling evidence of lithospheric pre-seismic ULF magnetic fields, but also evidence suggesting that these fields may have specific properties. To date, there's no comprehensive theory to explain the "observed" characteristics of ULF precursors and an attempt to this effect is reported herein. The basic model originates 
in earlier work by Vallianatos and Tzanis (1999b), which for the sake of completeness and comprehensiveness is also reported herein in some detail. The theory assumes that spontaneous electrification develops in an ensemble of crustal volumes (sources) within the seismogenic zone, which are distributed according to a fractal power law. The exact electrification mechanism does not have to be specified, so long as it allows for the development of a macroscopic field by the constructive superposition of the individual sources. On this basis, it is possible to show that the pre-seismic ULF magnetic field will be mainly vertical and to predict a scaling law between the amplitude of the vertical component and the magnitude of the expected earthquake. The scaling law, in turn, provides important clues as to the nature of the electrification process. Moreover, on the assumption that these sources emit incoherently, with relaxation times distributed according to an Arrhenius law, it is possible to explore the conditions under which the power spectrum will have a $1 / f$ spectral decay. The details of this analysis are presented in the following.

\section{Generation and scaling laws of pre-seismic ULF fields}

To begin with, for simplicity we consider spherical earthquake sources which include spherical electric emitters. However, as will easily be apparent, the theory may be directly generalized to sources with different shapes. Consider a sphere of radius $L$ and volume $V$, embedded in a conductive medium of constant resistivity $\rho$. Next, assume that the spherical volume acquires time dependent induced polarization $P(t)$ and becomes a source of electric and magnetic fields. For the moment, suppose that the polarization vector has only vertical component $P(t)=P(t) \hat{z}$ and that at $t=0$, there is a step change in polarization from zero to some finite value. Immediately after $t=0$, the potential $\Psi_{e}$ at an external point to the sphere will be given by (Griffiths, 1996),

$\Psi_{e}(t)=\frac{P(t) V \cos \theta}{4 \pi \varepsilon r^{2}}$ for $\mathrm{r}>\mathrm{L}$.

Since the sphere is embedded in a conducting medium, currents flow to reduce the surface charge $q_{A}(t)=P(t) \cdot \cos \theta$ and just outside the surface of the sphere, the normal current density $J_{n}(t)$ is

$J_{n}(t)=\frac{E_{n}(t)}{\rho}=\frac{2 P(t) \cos \theta}{3 \varepsilon \rho}=\frac{2 q_{A}(t)}{3 \varepsilon \rho}$

and $\quad J_{n}(t)=\frac{\partial q_{A}(t)}{\partial t}$

Hence, we conclude that

$\frac{\partial q_{A}(t)}{\partial t}+\frac{2 q_{A}(t)}{3 \varepsilon \rho}=0$,

which shows that the surface charge of a spherical object embedded in a conducting full-space will decay with a time constant equal to $3 \varepsilon \rho 2$. Introducing the frequency response indicated by (2) into (1) we obtain

$\Psi_{e}(\omega)=\frac{P(\omega) V \cos \theta}{4 \pi \varepsilon r^{2}}\left(\frac{i \omega}{i \omega+2 / 3 \varepsilon \rho}\right)$.

Herein we consider fields in the quasi-static approximation and may neglect the feedback from the magnetic field. Moreover, our result can be readily generalized for an arbitrary orientation of the polarization vector, in which case the electrostatic field becomes:

$\boldsymbol{E}(\omega)=-\nabla \Psi_{e}(\omega)=-\frac{V}{4 \pi \varepsilon} \cdot \frac{i \omega}{i \omega+2 / 3 \varepsilon \rho} \nabla\left(\frac{\boldsymbol{P}(\omega) \cdot \boldsymbol{r}}{r^{3}}\right)$.

Equation (4) has a corner frequency at $\omega_{c}=2 / 3 \varepsilon \rho$. For $\rho<10^{5} \Omega \mathrm{m}$ we find $\omega_{c}>120 \mathrm{kHz}$, well above the ULF frequency range. By taking the low frequency asymptote,

$\boldsymbol{E}(\omega)=-\frac{3 V \rho}{8 \pi} \cdot\left[i \omega \nabla\left(\frac{\boldsymbol{P}(\omega) \cdot \boldsymbol{r}}{r^{3}}\right)\right]$

and on transforming back to the time domain,

$\boldsymbol{E}(t)=\frac{3 V \rho}{8 \pi} \nabla\left(\frac{\dot{\boldsymbol{P}}(t) \cdot \boldsymbol{r}}{r^{3}}\right), \quad \dot{\boldsymbol{P}}=\frac{\partial \boldsymbol{P}(t)}{\partial t}$

A similar approach can be used for the magnetic field. From Maxwell's equations we have,

$\nabla \times \boldsymbol{B}(t)=\mu\left(\boldsymbol{J}(t)+\varepsilon \frac{\partial \boldsymbol{E}(t)}{\partial t}\right)=\mu\left(\frac{\boldsymbol{E}(t)}{\rho}+\varepsilon \frac{\partial \boldsymbol{E}(t)}{\partial t}\right)$.

On using Stokes' theorem and transforming the result to the frequency domain,

$\oint \boldsymbol{B}(\omega) \cdot d \ell=\mu \varepsilon\left(i \omega+\frac{1}{\rho \varepsilon}\right) \int_{S} \int \boldsymbol{E}(\omega) \cdot d S$.

An appropriate contour along which to compute the line integral is the perimeter of the surface $S$. The problem has spherical symmetry and the magnetic field lines form circles centred around the axis of symmetry and on a plane perpendicular to it. Thus, we can choose such a circle for the contour of integration, in which case $S$ is the enclosed disk. After some algebra the magnetic field $\boldsymbol{B}(\omega)$ is

$\boldsymbol{B}(\omega)=\frac{\mu}{4 \pi}\left[i \omega\left(i \omega+\frac{1}{\rho \varepsilon}\right) \cdot\left(i \omega+\frac{2}{3 \rho \varepsilon}\right)^{-1}\right] \cdot V \frac{\boldsymbol{P}(\omega) \times \boldsymbol{r}}{r^{3}}$.

By taking the low frequency asymptote again, we obtain

$\boldsymbol{B}(\omega)=\frac{3 \mu}{8 \pi} V \frac{i \omega \boldsymbol{P}(\omega) \times \boldsymbol{r}}{r^{3}}$,

which is easily transformed to the time domain to yield.

$\boldsymbol{B}(t)=\frac{3 \mu}{8 \pi} V \frac{\dot{\boldsymbol{P}}(t) \times \boldsymbol{r}}{r^{3}}$.

Recall that (6) and (11) are valid only for a polarized sphere in a conducting full-space. In order to estimate the field at 


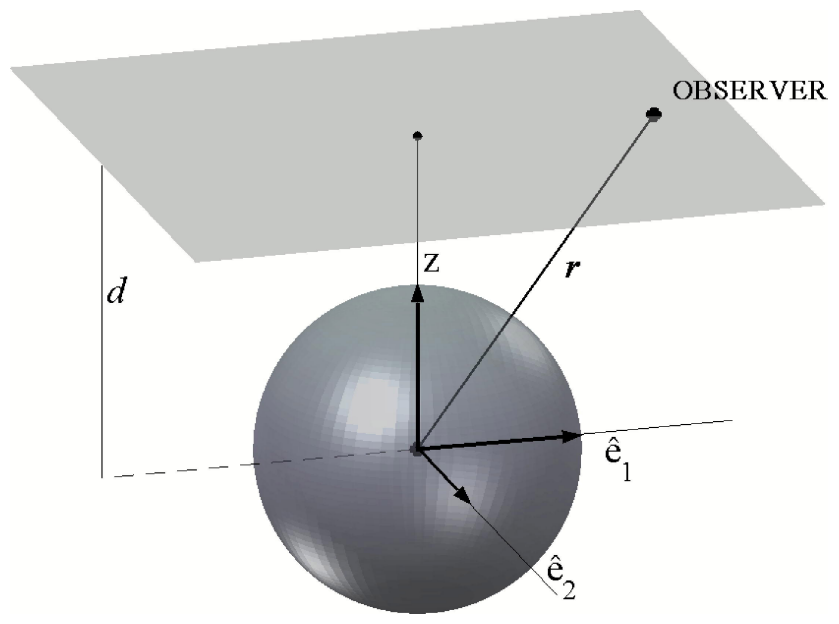

Fig. 1. Spherical co-ordinate system for computation of the electric field due to a polarised sphere in a conducting half-space.

the surface of the Earth (i.e. on the top of a conductive halfspace), we use image theory. We define a co-ordinate system with $\hat{z}$ being a unit vector in the vertical direction, and $\hat{\boldsymbol{e}}_{1}$ a unit vector perpendicular to $\hat{z}$, on the plane defined by the vectors $\hat{z}$ and $\boldsymbol{r}$ (Fig. 1). Then $\boldsymbol{P}(t)$ may be written as

$\boldsymbol{P}(t)=P_{z}(t) \hat{z}+P_{1}(t) \hat{\boldsymbol{e}}_{1}+P_{2}(t) \hat{\boldsymbol{e}}_{2}$,

where $P_{z}(t)$ is the vertical component of the polarization and $P_{1}(t), P_{2}(t)$ are the horizontal components in the sourcereceiver direction and perpendicular to it, respectively. Using the fields of the real and image sources we compute the magnetic field at the surface of the Earth, which turns out to be

$\boldsymbol{B}(t)=\frac{3 \mu V}{4 \pi r^{2}} \sin \theta \frac{\partial P_{2}(t)}{\partial t} \hat{z}$,

i.e. it is mainly vertical and observable only if the seismogenic process generates a source with polarization rate having a strong component perpendicular to the plane $(\hat{z}, \boldsymbol{r})$. This result is consistent with what is expected from the theory of magnetic fields from dc sources, which predicts that external to the earth magnetic fields can only be generated by subsurface current configurations with a significant horizontal component (for a comprehensive account see Edwards and Nabighian, 1991). It is also consistent with previous observations indicating that the precursory ULF magnetic data were overall vertically polarised.

Now, consider an earthquake source volume $V_{s}$ (again assumed spherical for simplicity), with radii $L_{s}$. Next, consider a set of distributed spherical sub-volumes $v_{i}$ with radius $l_{i}$ in $V_{s}$, which develop time dependent electrical polarization with geometry consistent over the entire set of sub-volumes. This comprises a set of constructively interfering EM field emitters. We introduce the working hypothesis that the number $N\left(l_{i}\right)$ of sub-volumes with radius $l_{i}$, is distributed according to a power law of the form

$N\left(l_{i}\right)=A \cdot l_{i}^{-D}$, where $0<D<3$. Then, the total volume of the spherical emitters is given by (Turcotte, 1997)

$V_{e}=\sum_{i} v_{i}=\frac{4 \pi}{3} \frac{A D}{3-D}\left(l_{\max }^{3-D}-l_{\min }^{3-D}\right)=\frac{4 \pi}{3} \frac{A D}{3-D} l_{\max }^{3-D} S_{R}$,

where $l_{\max }$ and $l_{\min }$ are the uppermost and lowermost radii sizes in the set $v_{i}$ and $S_{R}=1-\left(l_{\min } / l_{\max }\right)^{3-D}$ a scaling range factor $\left(0<S_{R}<1\right)$. It is expected that the upper limit $l_{\max }$ is a fraction $\kappa$ of the maximum size $L_{s}$ of the excited domain. Thus, we may assume $l_{\max } \approx \kappa L_{s}, 0 \ll \kappa<1$. Hence,

$V_{e}=\sum_{i} v_{i}=\frac{4 \pi}{3} \frac{A D}{3-D}\left(\kappa L_{s}\right)^{3-D} S_{R}$.

Under the condition $l_{\min } \ll l_{\max }$, it is straightforward to see that $S_{R} \approx 1$. The total vertical magnetic field observed at a distance $r$ from the emitters can be computed on the basis of the superposition principle and is

$B_{z}(t)=\left(\frac{\mu}{3-D} \frac{A D}{r^{2}} \kappa^{3-D} \sin \theta \frac{\partial P_{2}(t)}{\partial t}\right) L_{S}^{3-D}$.

The logarithm of Eq. (17) is

$\log B_{z}(t)=(3-D) \log L_{S}+$

$\log \left(\frac{\mu}{3-D} A D \kappa^{3-D} \sin \theta \frac{\partial P_{2}(t)}{\partial t}\right)-2 \log (r)$

whence, by virtue of the well known scaling relationship $\log \left(L_{S}\right)=0.5 M+$ Constant (e.g. Scholz, 1990),

$\log B_{z}(t)=\frac{3-D}{2} M+C_{S}^{B}(t)$.

Therefore, the pre-seismic vertical magnetic field scales with the magnitude with a universal slope of $\alpha=(3-D) / 2$. Note also that a scaling law with an identical universal slope has already been reported for the case of Electric Earthquake Precursors by Vallianatos and Tzanis (1999b).

Let us, now, investigate the source properties implied by Eqs. (17) and (19). Our model suggests that the ULF field is generated by a self-similar, hierarchical set of emitters. In the schizosphere and at the depths at which large earthquakes nucleate, the most common self-similar feature and omnipresent in the processes earthquake preparation are fractures and faults, which also obey rules of finite hierarchy. Therefore, the dependence of field strength on the fractal dimension implies a corresponding association of the electrification with fractures and faults (for a review see Tzanis and Vallianatos, 2001). This also justifies our assumption that $l_{\min } \ll l_{\max }$, because $l_{\min }$ would be the smallest fracture/crack size and $l_{\max }$ is comparable to the size of the rupture that will produce the main shock (also see Turcotte, 1997). A number of fragmentation experiments indicate that $2.2<D<2.8$ (e.g. see Table 3.2 of Turcotte, 1997), although deviations from this range have also been observed. However, the condition $D>2$ is necessary to constrain the total area of the fragments to a finite value. Observations 
of fault networks indicate that the two-dimensional fractal dimension $D_{2} \approx 1.6$, (e.g. Turcotte, 1997 and references therein). However, unfragmented blocks are bounded by micro- and macro-fractures and faults, so that a fractal distribution of block sizes in three dimensions can be related to the fractal distribution of fractures and faults in two-dimensions. This relationship is demonstrated by Turcotte (1997) on the basis of the comminution model of fragmentation, so that $D_{3}=D_{2}+1=2.6$. Termonia and Meakin (1986) simulated the growth of two-dimensional cracks using a kinetic fracture model and find $D_{2}=1.27$ for the surface topography, which can be generalized as above to $D_{3}=2.27$ and is consistent with the experimental results quoted therein. Hirata et al. (1987) produced explicit experimental results in granites showing that $D_{3}=2.75$ for transient creep, $D_{3}=2.66$ for steady creep and $D_{3}=2.25$ for acceleration creep. The latter corresponds to the phase of dynamic crack propagation (microfracturing) and clustering: as the creep progresses, the 3-D crack network becomes increasingly clustered and the fractal dimension decreases. Thus, we may assume that $\mathrm{D}$ varies in the range (2.25-2.6), taking the lower values during dynamic crack propagation. Accordingly, the slope $\alpha=(3-D) / 2$ varies in the range $(0.375-0.2)$. For $D \approx 2.3$ (tertiary creep) it is $\alpha \approx 0.35$, while for $D \approx 2.5$ (steady creep) $\alpha \approx 0.25$. Therefore, if the scaling law (19) is verified experimentally, in theory it should be possible to evaluate the state of the seismogenetic process by monitoring the development of the slope $\alpha(t)$.

\section{Spectral properties of pre-seismic ULF fields}

A number of studies (Hayakawa et al., 1996, 1999; Smirnova et al., 2001; Hattori et al., 2002) suggest that the power spectral density of the ULF signals decays with a power law form $1 / f^{\beta}$, which is characteristic of fractal time series, and that the exponent $\beta$ decreases toward a value of unity before the earthquake, which is characteristic of self-organized critical dynamics. One possible explanation for the $1 / f$ spectral decay and the SOC dynamics is that the observed ULF power spectrum results from the superposition of a large number of simultaneous sources with different relaxation times.

At ULF frequencies (quasi-static limit), displacement currents are negligible and the EM field propagates as a diffusion wave driven by the source current density $j_{s}(\boldsymbol{r}, t)$. Outside of the source, the diffusion of the current (and the EM field) will be appropriately described by an equation of the form:

$\frac{1}{\tau} \nabla j(\boldsymbol{r}, t)+\frac{d j(\boldsymbol{r}, t)}{d t}+\frac{1}{\tau} j(\boldsymbol{r}, t)=\frac{1}{\tau} j_{s}(\boldsymbol{r}, t)$

where $\tau$ is a relaxation time dependent on the properties of the material outside of the source. At any fixed point $\boldsymbol{r}$, the time-dynamics of this process will be

$\frac{d j(t)}{d t}+\frac{1}{\tau} j(t)=\frac{1}{\tau} J_{s}(t)$.

with $J_{s}(t)=\tau^{-1}\left[\left(j_{s}(\boldsymbol{r}, t)-\nabla j(\boldsymbol{r}, t)\right]\right.$ a generalized source function that includes the time dependent gradient. It is straightforward to conclude that the frequency dependence of such a process will be given by a Lorentz type power spectral density (PSD)

$s(\omega, \tau) \frac{\tau s_{j}(\omega)}{1+(\omega \tau)^{2}}$,

where $s_{J}(\omega)$ is the power spectrum density of $J_{S}(t)$ and may have its own frequency dependence.

Consider now, that due to the inhomogeneity of the seismic source region the relaxation time $\tau$ may not be unique and the same for all the EM field sources. Instead, it is more probable that there will be a spectrum of relaxation times dependent on activation energies $E$ required to drive the process. The relation between relaxation time and activation energy is the famous law of Arrhenius

$\tau=\tau_{0} \cdot \exp (U / k T)$,

where $T$ is the absolute temperature and $k$ is the Boltzmann constant (e.g. Dekker, 1971). Now, suppose for simplicity that the activation energies are distributed uniformly in the interval $\left[U_{1}, U_{2}\right]$. Then the distribution of the relaxation time $p(\tau)$ can be obtained by applying the rules of transforming probabilities, to yield a hyperbolic distribution for $\tau$, of the form

$p(\tau)=\frac{k T}{U_{1}-U_{2}} \frac{1}{\tau}, \quad \tau_{1} \leq \tau \leq \tau_{2}$

where $\tau_{1,2}=\tau_{0} \exp \left(E_{1,2} / k T\right)$.

The superposition of many independent relaxation processes with power spectra given by a Lorentz PSD and hyperbolically distributed relaxation times reads

$S(\omega)=\int_{\tau_{1}}^{\tau_{2}} p(\tau) \cdot s(\omega, \tau) d \tau$.

After some algebra,

$S(\omega)=\frac{k T s_{J}(\omega)}{2 \pi\left(U_{1}-U_{2}\right)} \frac{1}{f}\left[\arctan \left(\omega \tau_{2}\right)-\arctan \left(\omega \tau_{1}\right)\right]$.

In the frequency interval $1 / 2 \pi \tau_{1} \ll f \ll 1 / 2 \tau \pi_{2}$ (i.e. when $\left.\omega \tau_{2} \ll 1 \ll \omega \tau_{1}\right)$ we expand the inverse tangent terms inside the brackets after Abramowitz and Stegun (1970), to obtain:

$\arctan \left(\omega \tau_{2}\right) \approx \omega \tau_{2}-\frac{\left(\omega \tau_{2}\right)^{3}}{3}+\ldots$
$\arctan \left(\omega \tau_{1}\right) \approx \frac{\pi}{2}-\frac{1}{\omega \tau_{1}}+\frac{1}{3\left(\omega \tau_{1}\right)^{3}}+\ldots$

Under the condition $\omega \tau_{2} \ll 1 \ll \omega \tau_{1}$, it is easy to see that the difference inside the brackets, in spite of its somewhat awesome appearance, is roughly constant and varies slightly around the value of $-\pi / 2$. In consequence, the final expression for the PSD reduces to

$S(\omega) \approx \frac{k T s_{J}(\omega)}{4\left(U_{2}-U_{1}\right)} \frac{1}{f}$. 
Now, if $j_{s}(t)$ have time functions such as to generate evanescent diffusion waves with approximately white spectra $s_{J}(\omega)$ at ULF frequencies, then $S(\omega)$ will have an $1 / f$ spectral decay rate. This is possible when $j_{s}(\boldsymbol{r}, t)$ is relatively short in duration and not very far from the observer (for additional information and a more complete account see Vallianatos and Tzanis, 1999a and Tzanis and Vallianatos, 2002). If this theory is correct, it turns out that a $1 / f$ spectral decay requires almost continuous activity of many distributed sources, producing relatively short bursts of ULF emission. It should also be emphasized that the frequency interval where $1 / f$ dependence is observed could be quite broad. Suppose, for example, that the activation energies span a range as narrow as $7 k T$ (e.g. Dekker 1971), in which case $\tau_{2} / \tau_{1} \approx 10^{3}$. This would imply that the corresponding frequency band at which $S(\omega) \propto 1 / f$ extends over three orders of magnitude at least.

\section{Concluding remarks}

In this paper we attempt to explain some published observations of purported precursory ULF emissions by constructing a simple model of their sources. At the outset, we consider that the ULF source is a fractal set of crustal volumes, developing some precursory time-dependent electrical polarisation. The resulting magnetic field turns out to be mainly vertical and observable if the seismogenic process generates a source with polarisation rate perpendicular to the vertical plane through the source and the receiver. In addition, the amplitude of the pre-seismic vertical magnetic field scales linearly with the magnitude of the associated earthquake, with a constant universal slope dependent on the fractal dimension of the excited source region. Furthermore, assuming that the relaxations of the pre-seismic polarisation processes follow an Arrhenius law with uniformly distributed energies, we show that the macroscopic ULF field resulting from the superposition of such an ensemble of sources, may have a power spectrum decay proportional to $1 / f$ and exhibit self-organised critical dynamics.

The above ULF precursor model is general in the sense that it is independent of any particular electrification process and source power requirements. Moreover, it is reasonable in the sense that it builds on assumptions deriving from well understood physics and known properties of seismogenetic processes, such as is the fractal distribution of fracture-andfault networks and the SOC dynamics of their evolution. It does not need any other special conditions or particular earth structure. Nevertheless, reasonable as it may be, the model is still incomplete requiring further development and refinement. The question of whether it is also representative of the true physics of seismogenesis, is still pending.

On the other hand, the model is testable because it makes certain predictions of the expected properties of the ULF signal, which can be confirmed or refuted by observations. One such prediction is the amplitude-magnitude scaling law, which awaits confirmation. A second prediction, that the
ULF field is mainly vertical, has partially been confirmed, albeit retrospectively, by several observational studies, as discussed in the Introduction. Finally, the third prediction of the $1 / f$ spectral decay just prior to the earthquake also appears to be corroborated by a small number of observational studies, but is still far from having been confirmed. Most probably, it will take time, lots of work and tons of good luck before any definite answer can be given.

\section{References}

Abramowitz, M. and Stegun, I. A.: Handbook of Mathematical functions, Dover, 1970

Bak, P., Tang, C., and Wiesenfeld, K.: Self-Organized Criticality: An explanation of $1 / f$ noise, Phys. Rev. Lett., 59, 381-384, 1987.

Bak, P., Tang, C., and Wiesenfeld, K.: Self-Organized Criticality, Phys. Rev., 38, 364-374, 1988.

Dekker, A. J.: Solid State Physics, MacMillan, London, 1971

Dea, J. Y. and Boerner, W-M.: Observations of anomalous ULF signals preceding the Northridge earthquake of January 17, 1994, in: Atmospheric and Ionospheric Electromagnetic Phenomena Associated with Earthquakes, (Ed) Hayakawa, M., Terra Scientific Publishing Co, Tokyo, 137-145, 1999.

Edwards, R. N. and Nabighian, M. N.: The magnetometric resistivity method, SEG Series Investigations in Geophysics, 3, 2, 47104, 1991.

Fenoglio, M. A., Johnston, M. J. S., and Byerlee, J.: Magnetic and electric fields associated with changes in high pore pressure in fault zones - Application to the Loma Prietta ULF emissions, J. Geophys. Res., 100 (B7), 12 951-12 958, 1995.

Fraser-Smith, A. C., Bernardi, A., McGill P. R., Ladd, M. E., Helliwell, R. A., and Villard, O. G.: Low-frequency magnetic field measurements near the epicenter of the $M_{S}=7.1$ Loma Prieta Earthquake, Geophys. Res. Lett., 17, 1465-1468, 1990.

Griffiths, D. J.: Electrodynamics, 2nd Edition, Prentice Hall, 218223, 1996

Hattori, K., Akinaga, Y., Hayakawa, M., Yumoto, K., Nagao, T., and Uyeda, S.: ULF magnetic anomaly preceding the 1997 Kagoshima earthquake, in: Seismoelectromagnetics: Lithosphere-Atmosphere-Ionosphere Coupling, (Eds) Hayakawa, M. and Molchanov, O., Terra Sci. Pub. Co., Tokyo, 19-28, 2002.

Hayakawa, M. and Fujinawa, Y. (Eds): Electromagnetic Phenomena Related to Earthquake Prediction, Terra Sci. Pub. Co., Tokyo, 677 p., 1994.

Hayakawa, M., Kawate, R., Molchanov, O., and Yumoto, K.: Results of ultra-low-frequency magnetic field measurements during the Guam earthquake of 8 August 1993, Geophys. Res. Lett., 23 , 241-244, 1996.

Hayakawa, M. (Ed): Atmospheric and ionospheric electromagnetic phenomena associated with earthquakes, Terra Sci. Pub. Co., Tokyo, 996 p., 1999.

Hayakawa, M., Ito, T., and Smirnova, N.: Fractal analysis of ULF geomagnetic data associated with the Guam earthquake on $8 \mathrm{Au}-$ gust 1993, Geophys. Res. Lett., 26, 2797-2800, 1999.

Hayakawa, M. and Molchanov, O. A. (Eds): Seismo Electromagnetics: Lithosphere-Atmosphere-Ionosphere Coupling, Terra Scientific Publishing Co., Tokyo, 2002. 
Hirata, T., Satoh, T. and Ito, K.: Fractal structure of spatial distribution of microfracturing in rocks, Geophys. J. R. Astr. Soc., 107, 155-162, 1987.

Kawata, R., Molchanov, O., and Hayakawa, M.: Ultra low frequency magnetic fields during the Guam earthquake of $8 \mathrm{Au}-$ gust 1993 and their interpretation, Phys. Earth Planet. Inter., 105, 229-238, 1998.

Kopytenko, Y. A., Matiashvili, T., Voronov, P., Kopytenko, E., and Molchanov, O.: Detection of ultra-low-frequency emissions connected with the Spitak earthquake and its aftershock activity, based on geomagnetic pulsations data at Dusheti and Vardzia observatories, Phys. Earth Planet. Inter., 77, 85-95, 1993.

Kopytenko, Y. A., Ismagilov, V. S., Kopytenko, E., Voronov, P. and Petlenko, A. V.: Differential three-component ground-based measurements of magnetic fields varying in an ultralow frequency range, in: Electromagnetic Phenomena Related to Earthquake Prediction, (Eds) Hayakawa, M. and Fujinawa, Y., Terra Sci. Pub. Co., Tokyo, 247-252, 1994a.

Kopytenko, Y. A., Matiashvili, T., Voronov, P., and Kopytenko, E.: Observation of electromagnetic ultralow-frequency lithospheric emissions in the Caucasian seismically active zone and their connection with earthquakes, in: Electromagnetic Phenomena Related to Earthquake Prediction, (Eds) Hayakawa, M. and Fujinawa, Y., Terra Sci. Pub. Co., Tokyo, 175-180, 1994 b.

Molchanov, O. A., Kopytenko, Y. A., Voronov, P., Kopytenko, E., Matiashvili, T., Fraser-Smith, A. C., and Bernardi, A.: Results of ULF magnetic field measurements near the epicenters of the Spitak $\left(M_{S}=6.9\right)$ and Loma Prieta $(M=7.1)$ earthquakes: comparative analysis, Geophys. Res. Lett., 19, 1495-1498, 1992.

Park, S. K., Johnston, M. J. S., Madden, Th. R., Morgan, F. D., and Morrison, H. F.: Electromagnetic precursors to earthquakes in the ULF band: a review of observations and mechanisms, Rev.
Geophys., 31, 117-132, 1993.

Pilipenko, V. A., Fedorov, E. N., Yagova, N. V., and Yumoto, K.: Attempt to detect ULF electro-magnetic activity preceding earthquake, in: Atmospheric and Ionospheric Electromagnetic Phenomena Associated with Earthquakes, (Ed) Hayakawa, M., Terra Scientific Publishing Co, Tokyo, 203-214, 1999.

Scholz, C. H.: The Mechanics of Earthquakes and Faulting, Cambridge University Press, 1990.

Smirnova, N., Hayakawa, M., Gotoh, K., and Volobuev, D.: Scaling characteristics of ULF geomagnetic fields at the Guam seismoactive area and their dynamics in relation to the aerthquake, Nat. Haz. Earth Sys. Sci., 1, 3, 119-126, 2001.

Termonia, Y. and Meakin, P.: Formation of fractal cracks in a kinetic fracture model, Nature, 320, 429-431, 1986.

Turcotte, D. L.: Fractals and Chaos in Geology and Geophysics, Cambridge University Press, Second edition, 397 p., 1997.

Tzanis, A. and Vallianatos, F.: A critical review of ULF electric earthquake precursors, Annali di Geofisica, 44/2, 429-460, 2001.

Tzanis, A. and Vallianatos, F.: A physical model of electrical earthquake precursors due to crack propagation and the motion of charged edge dislocations, in: SeismoElectromagnetics: Lithosphere-Atmosphere-Ionosphere Coupling, (Eds) Hayakawa, M. and Molchanov, O. A., Terra Scientific Publishing Co., Tokyo, 117-130, 2002.

Vallianatos, F. and Tzanis, A.: A model for the generation of precursory electric and magnetic fields associated with the deformation rate of the earthquake focus, in: Seismic Atmospheric and Ionospheric electromagnetic Phenomena, (Ed) Hayakawa, M., Terra Scientific Publishing Co., Tokyo, 287-305, 1999a.

Vallianatos, F. and Tzanis, A.: On possible scaling laws between electric earthquake precursors (EEP) and earthquake magnitude, Geophys. Res. Lett., 26, 13, 2013-2016, 1999b. 